\title{
IDO expression in breast cancer: an assessment of 281 primary and metastatic cases with comparison to PD-L1
}

\author{
Erik A. Dill ${ }^{1}$ Patrick M. Dillon ${ }^{2} \cdot$ Timothy N. Bullock ${ }^{1}$ Anne M. Mills $\mathbb{1}^{1}$
}

Received: 18 January 2018 / Revised: 30 March 2018 / Accepted: 30 March 2018 / Published online: 25 May 2018

(c) United States \& Canadian Academy of Pathology 2018

\begin{abstract}
The immune inhibitory enzyme indoleamine 2,3-dioxygenase (IDO) has been associated with immune evasion in numerous malignancies and may mark these cancers as susceptible to anti-IDO therapies. We herein address IDO expression in breast cancers, examine the relationship between IDO and PD-L1, and investigate IDO fidelity across breast cancer primaries and metastases. IDO and PD-L1 expression was assessed in tissue microarrays containing 242 invasive primary breast cancers, 20 nodal metastases, and 19 distant metastases. IDO and PD-L1 were scored by extent in the tumor cells and immune infiltrate. Tumor IDO staining was seen in $14 \%$ of primaries including $38 \%$ of triple-negative cancers. IDO immune cell staining was seen in $14 \%$ of primaries and $29 \%$ of triple-negative cancers. Tumoral IDO and PD-L1 co-expression was seen in $8 \%$ of primaries, including $70 \%$ of tumoral PD-L1-positive cases. Immune IDO and PD-L1 co-expression was identified in $14 \%$ of primaries, including $48 \%$ of immune PD-L1-positive cases. Tumoral and immune cell IDO was conserved in $94 \%$ of matched primary/metastasis. In summary, IDO expression is common among high-grade, triple-negative breast cancers and is frequently associated with PD-L1 co-expression, suggesting that IDO might be a mechanism of anti-PD-1/PD-L1 immunotherapy resistance and that dual therapy may be of utility. Tumoral and immune cell IDO expression shows fidelity between primary and metastatic sites in treatment-naïve cancers, arguing against IDO as an independent driver for metastatic spread. Clinical trials are needed to assess the efficacy of IDO inhibition relative to IDO expression, as well as its possible role in combination with anti-PD-1/PD-L1 immunotherapy.
\end{abstract}

\section{Introduction}

The immune system is increasingly understood to play a critical role in the clearance, tolerance, and progression of a host of malignancies, including breast cancer. Studies have demonstrated that a robust cytotoxic T-cell response generally correlates with a favorable prognosis in breast carcinoma, whereas tumoral activation of the PD-1/PD-L1 checkpoint axis can allow tumors to evade that immune response [1-13]. Therapeutic inhibition of PD-1/PD-L1 has shown some promise for a subset of breast cancers, but has not proven uniformly effective [14-20]. The

Anne M. Mills

amm7r@virgnia.edu

1 University of Virginia Department of Pathology, Charlottesville, Virginia, USA

2 University of Virginia Department of Medicine, Division of Hematology \& Oncology, Charlottesville, Virginia, USA relatively limited success of anti-PD-1/PD-L1 therapies in breast cancer may stem in part from the fact that interference with this axis is only one of many ways a tumor responds to the host immune response. Indoleamine 2,3dioxygenase (IDO) is another immune modulatory molecule which may play a role in breast cancer prognosis and progression, and is of particular interest because it is clinically targetable [21-25].

IDO is an immunoregulatory enzyme that interferes with T-cell survival through depletion of tryptophan and generation of the toxic metabolite kynurenine. It normally plays an immunosuppressive role following inflammatory stimuli, preventing perpetual immune activation and associated pathology. However, as with checkpoint molecules such as PD-L1, malignancies may exploit the IDO pathway as a means of immune escape [23]. IDO expression has been identified in breast carcinomas and is particularly common among triple-negative, basal-like breast cancers [26]. This suggests that at least a subset of breast carcinomas may be vulnerable to drugs targeting IDO such as 1-methyl-DL-tryptophan, small molecule 
inhibitors such as Epacadostat, navoximod (GDC-0919), and BMS-986205, and IDO peptide vaccines [23, 27-29]. Immuno-oncology researchers are actively studying whether some IDO targeting approach may have utility as monotherapy or as adjuvants to other immunotherapies, including PD-1/PD-L1 checkpoint inhibitors [25]. However, the role of IDO inhibitors either alone or in combination with other therapies has yet to be fully elucidated in breast cancer, and IDO expression has not been well-studied across the full range of breast carcinoma subtypes.

We herein characterize IDO immunohistochemical expression across 242 cases of primary breast carcinoma and 39 cases of metastatic breast carcinoma representing a variety of subtypes, grades, and stages. Additionally, we evaluate the relationship between IDO and PD-L1 using data from a prior investigation of this case series [20].

\section{Materials and methods}

This study was approved by the institutional review board of The University of Virginia.

\section{Case selection and tissue microarray construction}

Cases were evaluated on four tissue microarrays containing archival formalin-fixed, paraffin-embedded tissues from 242 invasive primary breast carcinomas, 20 nodal metastases, and 19 distant metastases. Control tissues from kidney, liver, and placenta were also present. The arrays contained four replicate $0.6 \mathrm{~mm}$ cores from each case with samplings from different areas within the original tumor section including the tumor periphery and center.

The arrays were enriched to include a range of tumor stages and histologic subtypes. All primary tumors and all nodal metastases represented on the tissue microarrays were treatment-naïve. Sixteen of 19 distant metastases occurred after primary resection and therapy (mean time since primary diagnosis: 4.5 years), while 3 were identified at the time of primary diagnosis and were treatment-naïve. Sixteen of the nodal metastases and one lung metastasis were matched to concurrent primary tumors also present on the arrays; all matched cases were treatment naïve in both locales.

Pathologic variables including tumor histologic type, grade, stage, hormone receptor status, and mismatch repair status were obtained on all cases. $\mathrm{ER}^{+} / \mathrm{PR}^{+}$cases were classified as Luminal A-like, $\mathrm{ER}^{+} / \mathrm{PR}^{+} / \mathrm{HER} 2^{+}$cases were classified as Luminal B-like, hormone receptornegative cases with HER2 amplification were classified as $\mathrm{HER}^{+}{ }^{+}$, and hormone receptor/HER2 negative cases were classified as triple-negative. Formal expression profiling was not performed. BRCA mutation status was obtained when available.

\section{Immunohistochemistry}

Immunohistochemical staining for IDO (Sigma Prestige, HPA 023072, 1:2,000 dilution) and PD-L1/CD274 (Spring Biosciences, SP142, 1:200 dilution) was performed on all array slides using a Ventana Discovery Ultra and Leica Bond III, respectively. Importantly, the PD-L1 SP142 antibody clone has been validated on the Leica platform in our laboratory with $97 \%$ concordance to the FDA-approved Dako 22C3 antibody using the Dako platform. PD-L1 immunohistochemistry results were previously reported in detail in a prior study [20]. Both immunohistochemical stains were scored in both the tumor and the peritumoral immune compartment.

Tumor staining was classified as positive when clear staining was present in $\geq 1 \%$ of tumor cells. Cytoplasmic staining was considered positive for IDO, whereas only membranous staining was considered positive for PD-L1. Staining extent was further characterized in the following subcategories: $1-10,11-25,26-50$, and $>50 \%$. The $1 \%$ threshold for positivity was selected based on data demonstrating clinical response to PD-L1 inhibition at this expression level in some cancers, and percentage categories were further selected to include the cut-offs for all available PD-L1 inhibitors [7, 30]. Because there are no clinicallyaccepted thresholds for IDO expression, the same cut-points were used for this study.

Immune microenvironment staining was scored as positive when $>5 \%$ of peritumoral and intratumoral immune cells (including lymphocytes and macrophages) showed reactivity and was further subdivided by extent as 5-10, $11-25,26-50$, and $>50 \%$. The $5 \%$ lower limit was selected because single, scattered PD-L1 and IDO-positive inflammatory cells were observed in benign control tissues.

MMR protein immunohistochemistry was performed on all cases as part of a prior study [31]. MMR deficiency was diagnosed when there was complete loss of tumoral nuclear immunostaining for MLH1 (clone ES05, predilute; Leica Biosystems), PMS2 (clone MRG-28Mab, predilute; Cell Marque), MSH2 (clone 25D12, predilute; Leica), and/or MSH6 (clone $44 \mathrm{Mab}$, predilute; Cell Marque) in the setting of intact control stromal/lymphocyte staining. MMR protein loss identified on tissue microarray was confirmed on whole section slides.

\section{Statistical analysis}

The Fischer's exact test (two-tailed) was performed to assess the relationship between $B R C A$ status and IDO and PD-L1 expression (vassarstats.net). 
Fig. 1 Patterns of tumoral IDO expression. IDO expression was seen in $14 \%$ of primary breast cancers, including $38 \%$ of triplenegative carcinomas. Tumoral expression ranged from scattered single cell staining in $<10 \%$ of tumor cells (Case A/B; A: H\&E, B: IDO) to patchy staining involving $25-50 \%$ of cells (case C/D; C: H\&E, D: IDO) to diffuse staining involving $>50 \%$ of cells (case E/ F; E: H\&E; F: IDO)

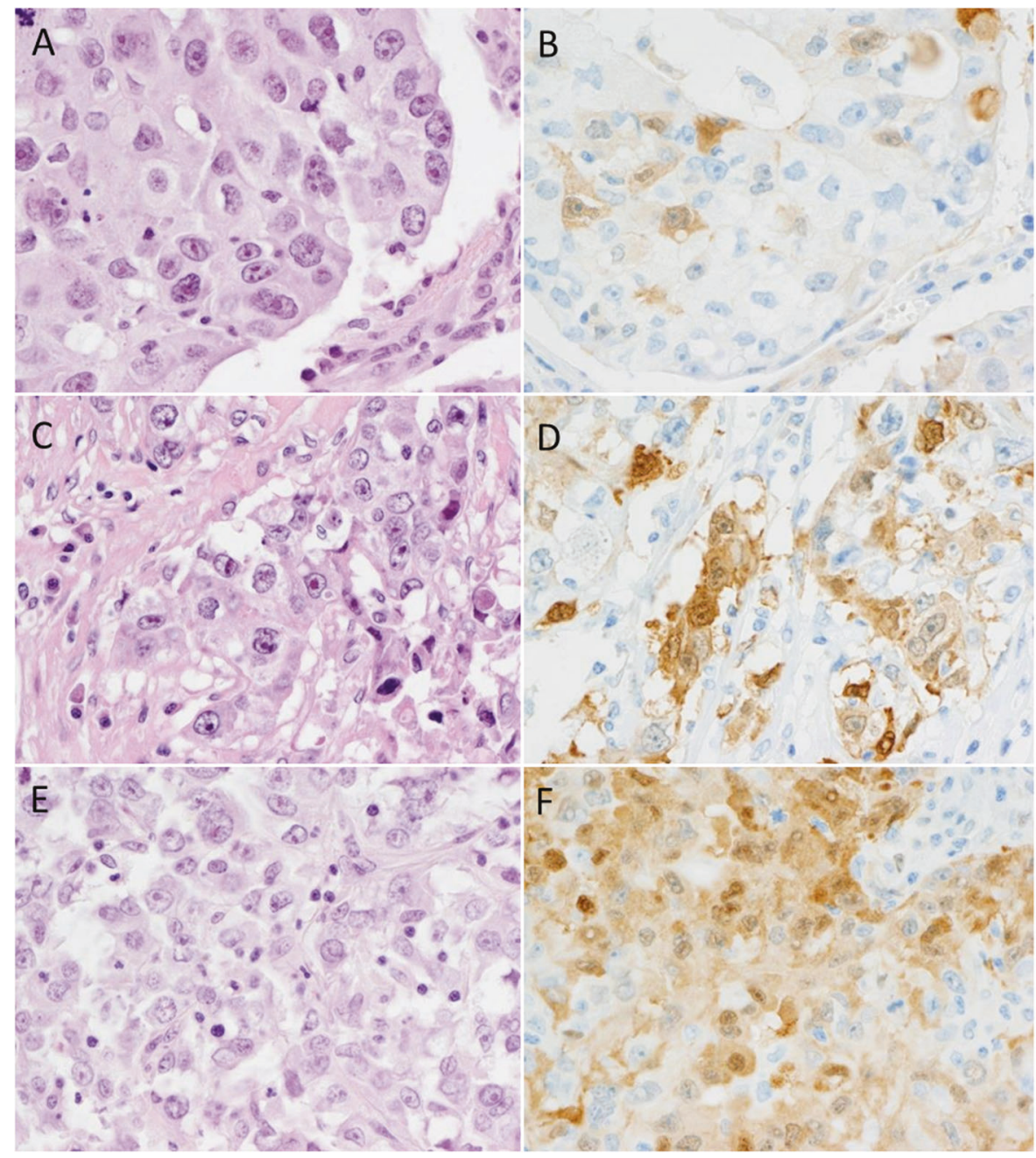

Table 1 Extent of IDO and PD-L1 staining in primary breast carcinomas

\begin{tabular}{lllll}
\hline Staining extent & Tumor IDO & Immune cell IDO & Tumor PD-L1 & Immune cell PD-L1 \\
\hline Negative (Tumor: <1\% Immune cell: $<5 \%)$ & $86 \%(208 / 242)$ & $86 \%(209 / 242)$ & $88 \%(215 / 245)$ & $71 \%(174 / 245)$ \\
$1-10 \%$ (Tumor) 5-10\% (Immune cell) & $8 \%(20 / 242)$ & $11 \%(27 / 242)$ & $4 \%(11 / 245)$ & $12 \%(29 / 245)$ \\
$11-25 \%$ (Tumor \& Immune cell) & $2 \%(4 / 242)$ & $2 \%(4 / 242)$ & $2 \%(6 / 245)$ & $9 \%(22 / 245)$ \\
$26-50 \%$ (Tumor \& Immune cell) & $2 \%(6 / 242)$ & $<1 \%(2 / 242)$ & $3 \%(7 / 245)$ & $6 \%(14 / 245)$ \\
$>50 \%$ (Tumor \& Immune cell) & $2 \%(4 / 242)$ & $0 \%(0 / 242)$ & $2 \%(6 / 245)$ & $2 \%(6 / 245)$ \\
\hline
\end{tabular}

\section{Results}

\section{IDO expression in primary tumors}

Tumor cell expression of IDO was observed in $14 \%$ of primary breast cancers. (Fig. 1) Most cases showed 1-10\% expression, while $4 \%$ had $>25 \%$ and $2 \%$ had $>50 \%$ tumoral positivity. (Table 1) Expression was frequently concentrated at the infiltrating edge of tumor nests, often in areas of high peritumoral lymphocyte density. The IDO-positive cases consisted predominantly of ductal cancers, including conventional ductal cases and those with medullary, apocrine, neuroendocrine, and metaplastic features. Expression in lobular cancers was rare. (Table 2A) Expression was most common in high-grade tumors with $37 \%$ of grade 3 , $3 \%$ of grade 2 , and $2 \%$ of grade 1 cancers showing tumoral staining. (Table 2B) IDO positivity was also most frequently observed in triple-negative cancers and HER2 ${ }^{+}$ cancers, with only $5 \%$ of Luminal A-like and Luminal Blike cases demonstrating expression. (Table 2C) Tumoral IDO expression was observed in $14 \%$ of $\mathrm{T} 1,15 \%$ of $\mathrm{T} 2,7 \%$ of $\mathrm{T} 3$, and $20 \%$ of $\mathrm{T} 4$ tumors. (Table $2 \mathrm{D}$ ) 
Table 2 IDO and PD-L1 expression in breast carcinomas*

A. IDO and PD-L1 expression in primary tumors by morphologic subtype

\begin{tabular}{|c|c|c|c|c|c|c|}
\hline Morphologic subtype & $\begin{array}{l}\text { Tumor IDO } \\
+\end{array}$ & $\begin{array}{l}\text { Immune cell } \\
\text { IDO+ }\end{array}$ & $\begin{array}{l}\text { Tumor PD-L1 } \\
+\end{array}$ & $\begin{array}{l}\text { Immune cell PD- } \\
\mathrm{L} 1+\end{array}$ & $\begin{array}{l}\text { Tumor IDO+ } \\
\text { PD-L1+ }\end{array}$ & $\begin{array}{l}\text { Immune Cell IDO+ } \\
\text { PD-L1+ }\end{array}$ \\
\hline Ductal, conventional & $\begin{array}{l}10 \%(16 / \\
156)\end{array}$ & $12 \%(19 / 156)$ & $8 \%(13 / 157)$ & $28 \%(44 / 157)$ & $4 \%(7 / 156)$ & $12 \%(19 / 156)$ \\
\hline Ductal with medullary features & $91 \%(10 / 11)$ & $82 \%(9 / 11)$ & $55 \%(6 / 11)$ & $100 \%(11 / 11)$ & $55 \%(6 / 11)$ & $82 \%(9 / 11)$ \\
\hline Ductal with apocrine features & $30 \%(3 / 10)$ & $10 \%(1 / 10)$ & $27 \%(3 / 11)$ & $36 \%(4 / 11)$ & $20 \%(2 / 10)$ & $10 \%(1 / 10)$ \\
\hline Lobular & $0 \%(0 / 23)$ & $4 \%(1 / 23)$ & $9 \%(2 / 23)$ & $13 \%(3 / 23)$ & $0 \%(0 / 23)$ & $4 \%(1 / 23)$ \\
\hline Pleomorphic lobular & $8 \%(1 / 13)$ & $0 \%(0 / 13)$ & $14 \%(2 / 14)$ & $14 \%(2 / 14)$ & $8 \%(1 / 13)$ & $0 \%(0 / 13)$ \\
\hline $\begin{array}{l}\text { Carcinoma with } \\
\text { Neuroendocrine features }\end{array}$ & $50 \%(1 / 2)$ & $100 \%(2 / 2)$ & $100 \%(2 / 2)$ & $100 \%(2 / 2)$ & $50 \%(1 / 2)$ & $100 \%(2 / 2)$ \\
\hline Micropapillary & $0 \%(0 / 2)$ & $0 \%(0 / 2)$ & $0 \%(0 / 2)$ & $0 \%(0 / 2)$ & $0 \%(0 / 2)$ & $0 \%(0 / 2)$ \\
\hline Metaplastic carcinoma & $60 \%(3 / 5)$ & $20 \%(1 / 5)$ & $40 \%(2 / 5)$ & $80 \%(4 / 5)$ & $40 \%(2 / 5)$ & $20 \%(1 / 5)$ \\
\hline Mucinous carcinoma & $0 \%(0 / 20)$ & $0 \%(0 / 20)$ & $0 \%(0 / 20)$ & $5 \%(1 / 20)$ & $0 \%(0 / 20)$ & $0 \%(0 / 20)$ \\
\hline \multicolumn{7}{|c|}{ B. IDO and PD-L1 expression in primary tumors by tumor grade } \\
\hline Grade & $\begin{array}{l}\text { Tumor IDO } \\
+\end{array}$ & $\begin{array}{l}\text { Immune cell } \\
\text { IDO+ }\end{array}$ & $\begin{array}{l}\text { Tumor PD-L1 } \\
+\end{array}$ & $\begin{array}{l}\text { Immune cell PD- } \\
\mathrm{L} 1+\end{array}$ & $\begin{array}{l}\text { Tumor IDO+ } \\
\text { PD-L1+ }\end{array}$ & $\begin{array}{l}\text { Immune cell IDO+ } \\
\text { PD-L1+ }\end{array}$ \\
\hline 1 & $2 \%(1 / 50)$ & $0 \%(0 / 50)$ & $4 \%(2 / 51)$ & $8 \%(4 / 51)$ & $0 \%(0 / 50)$ & $0 \%(0 / 50)$ \\
\hline 2 & $3 \%(3 / 111)$ & $5 \%(6 / 111)$ & $3 \%(3 / 112)$ & $14 \%(16 / 112)$ & $<1 \%(1 / 111)$ & $5 \%(6 / 111)$ \\
\hline 3 & $37 \%(30 / 81)$ & $33 \%(27 / 81)$ & $30 \%(25 / 82)$ & $62 \%(51 / 82)$ & $22 \%(18 / 81)$ & $33 \%(27 / 81)$ \\
\hline \multicolumn{7}{|c|}{ C. IDO and PD-L1 expression by ER/PR/HER2 status** } \\
\hline ER/PR/HER2 status & $\begin{array}{l}\text { Tumor IDO } \\
+\end{array}$ & $\begin{array}{l}\text { Immune cell } \\
\text { IDO+ }\end{array}$ & $\begin{array}{l}\text { Tumor PD-L1 } \\
+\end{array}$ & $\begin{array}{l}\text { Immune cell PD- } \\
\mathrm{L} 1+\end{array}$ & $\begin{array}{l}\text { Tumor IDO+ } \\
\text { PD-L1+ }\end{array}$ & $\begin{array}{l}\text { Immune cell IDO+ } \\
\text { PD-L1+ }\end{array}$ \\
\hline Luminal A-like $\left(\mathrm{ER}^{+} / \mathrm{PR}^{+}\right)$ & $5 \%(8 / 160)$ & $7 \%(11 / 160)$ & $5 \%(8 / 161)$ & $16 \%(26 / 161)$ & $3 \%(5 / 160)$ & $7 \%(11 / 160)$ \\
\hline $\begin{array}{l}\text { Luminal B-like }\left(\mathrm{ER}^{+} \mathrm{PR}^{+} \mathrm{HER} 2\right. \\
\left.{ }^{+}\right)\end{array}$ & $5 \%(1 / 20)$ & $5 \%(1 / 20)$ & $15 \%(3 / 20)$ & $35 \%(7 / 20)$ & $0 \%(0 / 20)$ & $10 \%(2 / 20)$ \\
\hline $\mathrm{HER}^{+}{ }^{+}\left(\mathrm{ER}^{-} \mathrm{PR}^{-} \mathrm{HER} 2^{+}\right)$ & $33 \%(1 / 3)$ & $33 \%(1 / 3)$ & $50 \%(2 / 4)$ & $50 \%(2 / 4)$ & $33 \%(1 / 3)$ & $33 \%(1 / 3)$ \\
\hline Triple-negative (ER ${ }^{-} \mathrm{PR}^{-} \mathrm{HER} 2^{-}$) & $38 \%(21 / 56)$ & $29 \%(16 / 56)$ & $32 \%(18 / 57)$ & $61 \%(35 / 57)$ & $21 \%(12 / 56)$ & $29 \%(16 / 56)$ \\
\hline \multicolumn{7}{|c|}{ D. IDO and PD-L1 expression by stage*** } \\
\hline Stage & $\begin{array}{l}\text { Tumor IDO } \\
+\end{array}$ & $\begin{array}{l}\text { Immune cell } \\
\text { IDO+ }\end{array}$ & $\begin{array}{l}\text { Tumor PD-L1 } \\
+\end{array}$ & $\begin{array}{l}\text { Immune cell PD- } \\
\mathrm{L} 1+\end{array}$ & $\begin{array}{l}\text { Tumor IDO+ } \\
\text { PD-L1+ }\end{array}$ & $\begin{array}{l}\text { Immune cell IDO+ } \\
\text { PD-L1+ }\end{array}$ \\
\hline $\mathrm{T} 1$ & $\begin{array}{l}14 \%(20 / \\
140)\end{array}$ & $11 \%(16 / 140)$ & $13 \%(18 / 143)$ & $25 \%(36 / 143)$ & $9 \%(12 / 140)$ & $11 \%(16 / 140)$ \\
\hline $\mathrm{T} 2$ & $15 \%(12 / 78)$ & $21 \%(16 / 78)$ & $14 \%(11 / 78)$ & $37 \%(29 / 78)$ & $8 \%(6 / 78)$ & $21 \%(16 / 78)$ \\
\hline T3 & $7 \%(1 / 15)$ & $7 \%(1 / 15)$ & $0 \%(0 / 15)$ & $13 \%(2 / 15)$ & $0 \%(0 / 15)$ & $7 \%(1 / 15)$ \\
\hline $\mathrm{T} 4$ & $20 \%(1 / 5)$ & $0 \%(0 / 5)$ & $20 \%(1 / 5)$ & $20 \%(1 / 5)$ & $20 \%(1 / 5)$ & $0 \%(0 / 5)$ \\
\hline $\mathrm{N}+$ & $5 \%(1 / 20)$ & $5 \%(1 / 20)$ & $10 \%(2 / 20)$ & $50 \%(10 / 20)$ & $0 \%(0 / 20)$ & $5 \%(1 / 20)$ \\
\hline $\mathrm{M}+$ & $0 \%(0 / 19)$ & $11 \%(2 / 19)$ & $10 \%(2 / 20)$ & $50 \%(10 / 20)$ & $0 \%(0 / 19)$ & $11 \%(2 / 19)$ \\
\hline
\end{tabular}

*Analysis reflects 4 cases which were cut through between PD-L1 and IDO TMAs ( 3 primaries and 1 met)

**A Analysis excludes 3 primaries for which hormone receptor/HER2 status was unknown

****Analysis excludes 4 primaries for which stage was unknown

\section{IDO expression in tumor-associated immune cells}

Tumor-associated inflammatory cell expression of IDO was observed in $14 \%$ of primary breast cancers. (Fig. 2) Most cases showed $5-10 \%$ expression, while $<1 \%$ had $>25 \%$ and $0 \%$ had $>50 \%$ tumoral positivity. (Table 1 ) The IDO positive cases spanned a range of histologies paralleling those observed in the tumoral staining group. (Table 2.A) IDO immune cell positivity was most common in high-grade tumors, 33\% of which showed staining. (Table 2.B) IDO positivity was also most frequently observed in triple-negative cancers and $\mathrm{HER}^{+}$cancers, with only occasional 
Fig. 2 Patterns of immune IDO expression. Tumor-associated inflammatory cells expressed IDO in $14 \%$ of primary breast cancers, including $29 \%$ of triplenegative carcinomas. Immune cell staining was predominantly identified in lymphocytes intimately admixed with the tumor cells. While occasional cases showed IDO staining in over half of tumor-associated immune cells (case A/B; A: $\mathrm{H} \& \mathrm{E}, \mathrm{B}$ : IDO), patchy staining involving $<10 \%$ of the inflammatory component was more common (case C/D; C: H\&E; D: IDO)

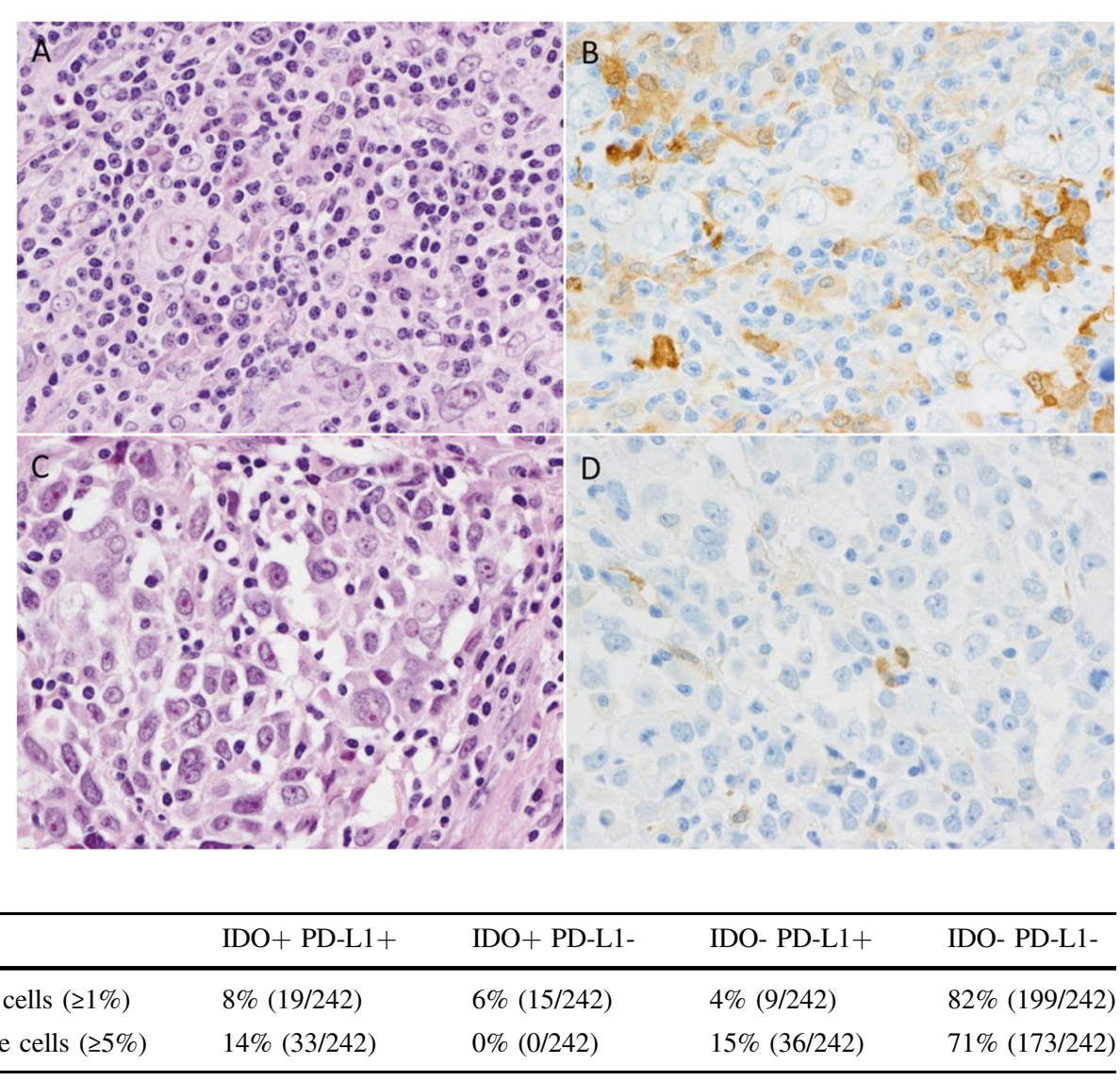

\begin{tabular}{lllll}
\hline & IDO+ PD-L1+ & IDO+ PD-L1- & IDO- PD-L1+ & IDO- PD-L1- \\
\hline Tumor cells $(\geq 1 \%)$ & $8 \%(19 / 242)$ & $6 \%(15 / 242)$ & $4 \%(9 / 242)$ & $82 \%(199 / 242)$ \\
Immune cells $(\geq 5 \%)$ & $14 \%(33 / 242)$ & $0 \%(0 / 242)$ & $15 \%(36 / 242)$ & $71 \%(173 / 242)$ \\
\hline
\end{tabular}

Table 3 Co-expression of IDO and $\mathrm{PD}-\mathrm{L} 1$ in primary breast carcinomas
Luminal A-like and Luminal B-like cases demonstrating expression within the tumor-associated inflammation. (Table 2.C) Immune cell expression was observed in $11 \%$ of $\mathrm{T} 1,21 \%$ of $\mathrm{T} 2$, and $7 \%$ of $\mathrm{T} 3$; while no $\mathrm{T} 4$ cases demonstrated positivity. (Table 2.D)

\section{IDO expression in metastases}

Tumor cell expression of IDO was observed in 5\% of nodal and $0 \%$ of distant metastases. (Table 2D) Of the matched treatment-naïve primary and metastatic tumors, 94\% showed concordant staining between the primary and metastatic tumor, with one case demonstrating loss of IDO positivity from the primary to the metastatic tumor.

Immune cell expression of IDO was observed in 5\% of nodal and $11 \%$ of distant metastases (Table 2D). Ninetyfour percent showed concordant staining between the primary and metastatic tumor-associated inflammatory cells, with one case demonstrating loss of IDO positivity from the primary to the metastatic tumor.

\section{IDO and PD-L1 co-expression}

PD-L1 immunohistochemical results were provided in detail in a prior publication [20] and are summarized in
Table 2. Tumoral IDO and PD-L1 co-expression was seen in $8 \%$ of all primaries, which constituted $70 \%$ of all tumoral PD-L1-positive cases. (Table 3, Figs. 3 and 4) Of these 19 cases showing co-expression, $95 \%$ were grade 3 and $67 \%$ were triple-negative. By histomorphology $37 \%$ were conventional ductal while $32 \%$ exhibited medullary features. Among cases lacking tumoral expression of PD-L1, 93\% were also negative for IDO. Immune cell IDO and PD-L1 co-expression was identified in $14 \%$ of primaries, comprising $48 \%$ of all immune PD-L1-positive cases. Of these 33 cases showing co-expression, the majority (82\%) were grade 3 . By hormone receptor status, $53 \%$ were triplenegative while $37 \%$ were Luminal-A. By histomorphology, $58 \%$ were conventional ductal and $27 \%$ exhibited medullary features. All cases lacking immune cell expression of PD-L1 were also negative for IDO.

\section{IDO and PD-L1 expression, mismatch repair status, and germline BRCA status}

A single case showed mismatch repair (MLH1/PMS2) loss confirmed on whole tissue sections. This tumor expressed IDO and PD-L1 in a subset $(<10 \%)$ of tumor cells and showed diffuse PD-L1 immune cell staining with only focal IDO staining. Cases from BRCA-mutated patients showed a trend towards increased tumoral IDO expression when 


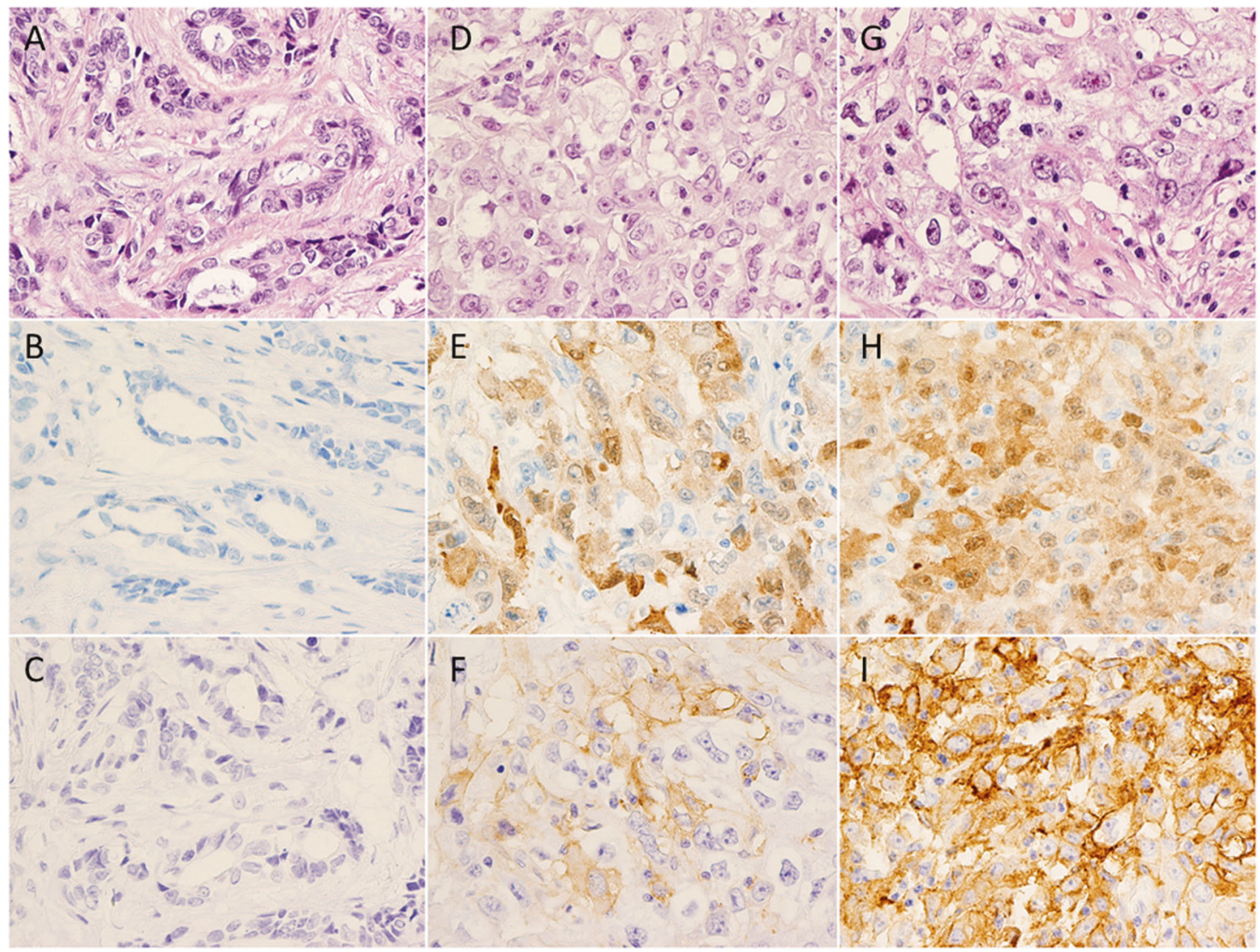

Fig. 3 IDO and PD-L1 co-expression. While the majority of cancers were negative for both IDO and PD-L1 (Case A-C; A: H\&E, B: IDO; C: PD-L1), $11 \%$ of tumors co-expressed these immune modulatory molecules. Co-expression was most common among triple-negative

compared to BRCA-wildtype patients; however, this trend was not statistically significant $[67 \%(4 / 6)$ vs. $25 \%(4 / 16)$, $p=0.137]$ and numbers were limited. No significant differences were observed for immune IDO or immune/ tumoral PD-L1 expression between BRCA-mutated and wildtype patients.

\section{Discussion}

Immune evasion plays a critical role in the progression of malignancy, and studies in a variety of cancers suggest that dysregulated IDO expression may be instrumental in facilitating some tumors' growth [32-35]. IDO was first discovered on placental vasculature and trophoblasts where it promotes maternal-fetal tolerance through local suppression of maternal $\mathrm{T}$ lymphocytes [36]. Murine models have shown that IDO can block clonal T-cell expansions and that loss of IDO contributes to a variety of autoimmune pathologies [37-41]. While these functions are ordinarily important for maintaining a healthy immune balance, IDO also promotes pathology when co-opted by cancer as an adaptive method of immune evasion [21, 23, 33, 42, 43]. cancers; co-expression patterns ranged from patchy tumoral positivity for both markers (case D-F; D: H\&E, E: IDO, F: PD-L1) to diffusely positive for both markers (case G-I; G: H\&E, H: IDO, I: PD-L1)

IDO has been identified in a variety of malignancies [32, 34, 42, 44-46]. Early evidence from non-small cell lung carcinomas has shown only rare co-expression of IDO and PD-L1 [34]. In the lower gastrointestinal tract and endometrium, IDO expression is most common among mismatch repair-deficient cancers, many of which co-express PD-L1 [32]. Mismatch repair deficiency is exceedingly uncommon among breast carcinomas, however, and has not been reproducibly linked to PD-L1 expression in this locale where other mechanisms, such as $B R C A$ mutations, are thought to be more important drivers of neoantigen production and immunogenicity [31]. Evidence from highgrade serous ovarian cancers suggests that $B R C A$ mutations are affiliated with higher mutation burdens, elevated T-cell infiltrates, and increased PD-L1 expression [47]. Recent work in breast carcinomas has shown that basal-like cancers are particularly likely to express IDO [25, 26]. Finally, while other studies have demonstrated PD-L1 expression in up to half of triple-negative breast carcinomas [14, 16, 20, $48,49]$, the relationship between IDO and PD-L1 in these cancers has not been well-investigated.

Our series adds to the literature on IDO in breast cancers and provides insight into the relationship between IDO and 
Fig. 4 IDO and PD-L1 marginal expression pattern. Positivity for both IDO and PD-L1 was often concentrated at the infiltrating edge of the tumor in regions of lymphocyte concentration, as illustrated in this high-grade triple-negative carcinoma (A: H\&E, B-C: IDO, D-E: PD-L1). This pattern is suggestive of an adaptive immune response

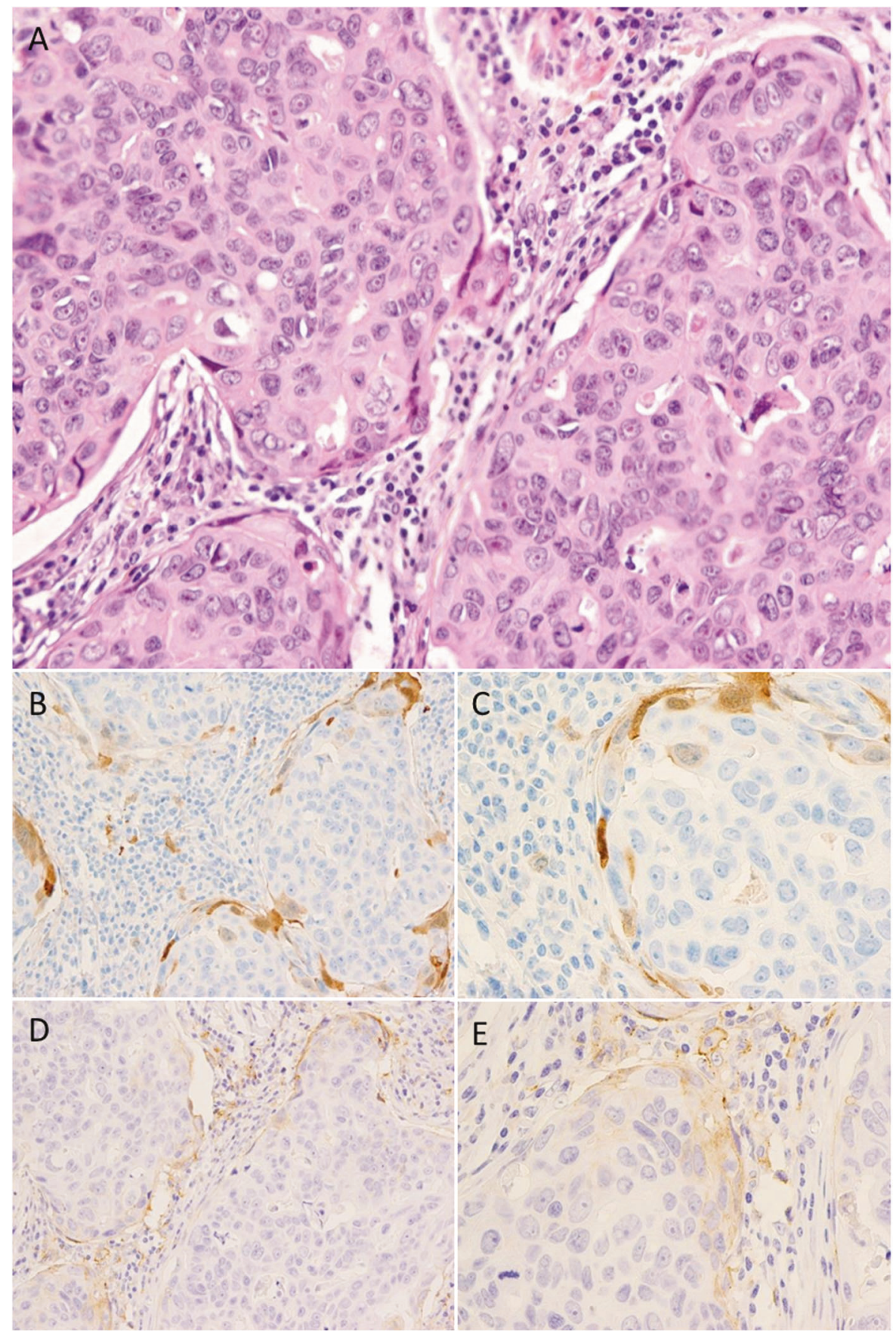

PD-L1 expression. The results corroborate prior work demonstrating that IDO expression is highest among triplenegative breast cancers. Additionally, we demonstrate that expression is not unique to basal-like cancers and can also be seen among triple-negative tumors with metaplastic and apocrine morphologies as well as a subset of HER2-positive and occasional hormone receptor-positive cancers. We also show a trend towards increased tumoral expression among $B R C A$-mutated patients; however, significance is limited by low numbers of patients with known BRCA status.
Although a prior study suggested a role for IDO in metastasis [50], our series demonstrates consistent IDO expression when comparing primary tumor and metastatic site biopsies among treatment-naïve patients, thus providing no evidence that metastatic spread is achieved through acquisition of IDO expression. However, the sample size of paired primaries and metastases is limited, therefore additional study may be warranted, as are investigations of the role of neoadjuvant therapy on expression status. 
Finally, we demonstrate that the majority $(70 \%)$ of PDL1-positive breast cancers co-express IDO. This finding may help account for variable responses to PD-1/PD-L1 inhibition even in the setting of strong PD-L1 expression, as efficacy of PD-1/PD-L1 antagonism could be limited in a microenvironment with IDO-associated T-cell impairment. This suggests a role for combination immunotherapy in select patients. Additionally, we show that the majority of cases lacking PD-L1 are also IDO-negative. Given the very low frequency of IDO-positive, PD-L1negative cases, PD-L1 status could potentially drive an algorithmic approach to IDO testing: if PD-L1 is expressed, the patient may benefit combination therapy and thus merit IDO staining; if PD-L1 is not expressed, however, IDO is also likely to be negative and there is little rationale for targeting either molecule.

However, it is not yet clear that IDO expression corresponds with response to anti-IDO therapy. Clinical trials correlating therapeutic response with immunohistochemical expression of IDO in the tumoral and immune compartments are therefore needed, as this biomarker may prove important for rational patient selection. There are a number of ongoing clinical trials investigating IDO inhibitors in breast cancer, but none have evaluated biomarker expression as an inclusion criterion. Recent phase I trials have focused on indoximod (1-methyl tryptophan) which has shown hints of single agent activity in breast cancer [51] and some efficacy in combination with docetaxel in breast cancers (NCT01792050) [52]. Other IDO inhibitors under phase I investigation include BMS-986205, GDC0919 [29], and epacadostat [53]. Vaccine trials of indoximod given in combination with p53 antigen are also underway (NCT01042535) [54]. Several studies have also focused on IDO inhibitors in combination with PD-1/PDL1 therapy: the ECHO202 study of epacadostat plus pembrolizumab in triple-negative breast cancer showed a response rate of $10 \%$ along with a $36 \%$ disease control rate [55], while trials of BMS-986205 plus nivolumab (NCT02658890), and GDC-0919 plus atezolizumab (NCT02471846) are ongoing.

As data emerge from these trials, it will be worthwhile to retrospectively assess IDO expression among responders. Our findings suggest that therapies targeting IDO are unlikely to show great utility in unselected populations of breast cancer patients since the majority of tumors do not appear to enlist this mechanism of immune evasion. Conversely, these drugs may benefit a specific subset of patients, most notably the roughly one-third of triplenegative cancers expressing IDO. Given that the majority of PD-L1-positive tumors co-express IDO, correlation with IDO and PD-L1 levels for the combination therapy trials will be of particular interest. Establishing tumoral and/or immune cell staining thresholds indicative of probable response will maximize the potential benefit of these drugs and spare patients unlikely to respond from unnecessary side effects.

A significant limitation of this approach-which is central to both our current study and to similar investigations in the immunotherapy literature-is that IDO is only one of many immune modulatory molecules that can be manipulated by malignancy. Though we herein describe IDO in relation to PD-L1 expression, there are many other immune modulatory molecules which may play a role in breast cancer progression and could further confound discordance between the expression status and therapeutic response. These two molecules are of interest because clinically available inhibitors exist, but as more targets emerge a more complete understanding of the immune milieu will help inform a rational approach to therapy. While immunohistochemical staining benefits from easy applicability to the clinical setting, more detailed multiplex investigations could present a more thorough picture of the challenges that targeted immunotherapies are up against and better highlight potential tumoral vulnerabilities.

Another significant limitation derives from the tissue microarray-based nature of this study. Immune modulatory molecules often show considerable geographic heterogeneity and although sampling errors were mitigated by the use of four replicate cores per case and attempted sampling at both the 'leading edge' and in the center of each, the potential under or overrepresentation of tumor characteristics remains a limitation. Whole section studies on breast cancers are therefore warranted, with particular attention to the complete tumor margin where the adaptive immune response is often heightened.

In summary, we herein demonstrate that IDO expression is frequent among high-grade, triple-negative breast carcinomas with less common expression among lowgrade, hormone receptor-positive cancers. The majority of cases with tumoral PD-L1 positivity co-express IDO, suggesting that IDO may play a role in anti-PD-1/PD-L1 immunotherapy resistance and that dual therapy may be of utility in this setting. Tumoral and stromal immune cell IDO expression shows fidelity between primary and metastatic sites in treatment-naïve cancers, arguing against IDO as an independent driver for metastatic spread. Clinical trials are needed to assess the efficacy of IDO inhibition relative to IDO expression, as well as its possible role in combination with anti-PD-1/PD-L1 immunotherapy.

Acknowledgements The authors would like to acknowledge the skill and expertise of the University of Virginia Biorepository and Tissue Research Facility in the construction of Tumor Microarrays. 


\section{Compliance with ethical standards}

Conflict of interest towards increased tumoral expressioPMD participates in trials sponsored by BMS, Pfizer, Lilly, Merck, Newlink, Abbvie, Novartis, Seattle Genetics and Genentech.The remaining authors declare that they have no conflict of interest.

\section{References}

1. Butte MJ, Keir ME, Phamduy TB, Sharpe AH, Freeman GJ. Programmed death-1 ligand 1 interacts specifically with the B7-1 costimulatory molecule to inhibit $\mathrm{T}$ cell responses. Immunity. 2007;27:111-22.

2. Dong H, Strome SE, Salomao DR, et al. Tumor-associated B7-H1 promotes T-cell apoptosis: a potential mechanism of immune evasion. Nat Med. 2002;8:793-800.

3. Brahmer JR, Tykodi SS, Chow LQ, et al. Safety and activity of anti-PD-L1 antibody in patients with advanced cancer. N Engl J Med. 2012;366:2455-65.

4. Becht E, Giraldo NA, Germain C, et al. Immune contexture, immunoscore, and malignant cell molecular subgroups for prognostic and theranostic classifications of cancers. Adv Immunol. 2016;130:95-190.

5. Ali HR, Provenzano E, Dawson SJ, et al. Association between CD8 + T-cell infiltration and breast cancer survival in 12,439 patients. Ann Oncol. 2014;25:1536-43.

6. Park JJ, Omiya R, Matsumura Y, et al. B7-H1/CD80 interaction is required for the induction and maintenance of peripheral $\mathrm{T}$-cell tolerance. Blood. 2010;116:1291-8.

7. Topalian SL, Hodi FS, Brahmer JR, et al. Safety, activity, and immune correlates of anti-PD-1 antibody in cancer. N Engl J Med. 2012;366:2443-54.

8. Tumeh PC, Harview CL, Yearley JH, et al. PD-1 blockade induces responses by inhibiting adaptive immune resistance. Nature. 2014;515:568-71.

9. Iwai Y, Ishida M, Tanaka Y, Okazaki T, Honjo T, Minato N. Involvement of PD-L1 on tumor cells in the escape from host immune system and tumor immunotherapy by PD-L1 blockade. Proc Natl Acad Sci Usa. 2002;99:12293-7.

10. Hughes PE, Caenepeel S, Wu LC. Targeted therapy and checkpoint immunotherapy combinations for the treatment of cancer. Trends Immunol. 2016;37:462-76.

11. Wang X, Teng F, Kong L, Yu J. PD-L1 expression in human cancers and its association with clinical outcomes. Onco Targets Ther. 2016;9:5023-39.

12. Zou W, Chen L. Inhibitory B7-family molecules in the tumour microenvironment. Nat Rev Immunol. 2008;8:467-77.

13. Reiss KA, Forde PM, Brahmer JR. Harnessing the power of the immune system via blockade of PD-1 and PD-L1: A promising new anticancer strategy. Immunotherapy. 2014;6:459-75.

14. Cimino-Mathews A, Thompson E, Taube JM, et al. PD-L1 (B7-H1) expression and the immune tumor microenvironment in primary and metastatic breast carcinomas. Hum Pathol. 2016;47:52-63.

15. Engel JB, Honig A, Kapp M, et al. Mechanisms of tumor immune escape in triple-negative breast cancers (TNBC) with and without mutated BRCA 1. Arch Gynecol Obstet. 2014;289:141-7.

16. Mittendorf EA, Philips AV, Meric-Bernstam F, et al. PD-L1 expression in triple-negative breast cancer. Cancer Immunol Res. 2014;2:361-70.

17. Nanda R, Chow LQ, Dees EC, et al. Pembrolizumab in patients with advanced triple-negative breast cancer: phase ib KEYNOTE012 study. J Clin Oncol. 2016;34:2460-7.

18. Wang M, Zhang C, Song Y, et al. Mechanism of immune evasion in breast cancer. Onco Targets Ther. 2017;10:1561-73.
19. Chawla A, Philips AV, Alatrash G, Mittendorf E. Immune checkpoints: a therapeutic target in triple negative breast cancer. Oncoimmunology. 2014;3:e28325.

20. Dill EA, Gru AA, Atkins KA, et al. PD-L1 expression and intratumoral heterogeneity across breast cancer subtypes and stages: an assessment of 245 primary and 40 metastatic tumors. Am J Surg Pathol. 2017;41:334-42.

21. Munn DH, Mellor AL. IDO in the tumor microenvironment: Inflammation, counter-regulation, and tolerance. Trends Immunol. 2016;37:193-207.

22. Munn DH, Mellor AL. Indoleamine 2,3 dioxygenase and metabolic control of immune responses. Trends Immunol. 2013;34: 137-43.

23. Moon YW, Hajjar J, Hwu P, Naing A. Targeting the indoleamine 2,3-dioxygenase pathway in cancer. $\mathrm{J}$ Immunother Cancer. 2015;3:51-015-0094-9. eCollection 2015

24. Isla Larrain MT, Rabassa ME, Lacunza E, et al. IDO is highly expressed in breast cancer and breast cancer-derived circulating microvesicles and associated to aggressive types of tumors by in silico analysis. Tumour Biol. 2014;35:6511-9.

25. Soliman H, Rawal B, Fulp J, et al. Analysis of indoleamine 2-3 dioxygenase (IDO1) expression in breast cancer tissue by immunohistochemistry. Cancer Immunol Immunother. 2013;62: 829-37.

26. Kim S, Park S, Cho MS, Lim W, Moon BI, Sung SH. Strong correlation of indoleamine 2,3-dioxygenase 1 expression with basal-like phenotype and increased lymphocytic infiltration in triple-negative breast cancer. J Cancer. 2017;8:124-30.

27. Jochems C, Fantini M, Fernando RI, et al. The IDO1 selective inhibitor epacadostat enhances dendritic cell immunogenicity and lytic ability of tumor antigen-specific $\mathrm{T}$ cells. Oncotarget. 2016;7:37762-72.

28. Blocking IDO1 helps shrink bladder, cervical tumors. Cancer Discov. 2018;8:OF3.

29. Nayak A, Hao Z, Sadek R, Dobbins R, Marshall L, et al. Phase 1a study of the safety, pharmacokinetics, and pharmacodynamics of GDC-0919 in patients with recurrent/advanced solid tumors. Eur J Cancer. 2015;51:S69.

30. Taube JM, Klein A, Brahmer JR, et al. Association of PD-1, PD-1 ligands, and other features of the tumor immune microenvironment with response to anti-PD-1 therapy. Clin Cancer Res. 2014;20:5064-74.

31. Mills AM, Dill EA, Moskaluk CA, Dziegielewski J, Bullock TN, Dillon PM. The relationship between mismatch repair deficiency and PD-L1 expression in breast carcinoma. Am J Surg Pathol. 2017;42:183-91.

32. Friedman K, Brodsky AS, Lu S, et al. Medullary carcinoma of the colon: a distinct morphology reveals a distinctive immunoregulatory microenvironment. Mod Pathol. 2016;29:528-41.

33. Iversen TZ, Andersen MH, Svane IM. The targeting of indoleamine 2,3 dioxygenase-mediated immune escape in cancer. Basic Clin Pharmacol Toxicol. 2015;116:19-24.

34. Schalper KA, Carvajal-Hausdorf D, McLaughlin J, et al. Differential expression and significance of PD-L1, IDO-1, and B7-H4 in human lung cancer. Clin Cancer Res. 2017;23:370-8.

35. Sordillo PP, Sordillo LA, Helson L. The kynurenine pathway: a primary resistance mechanism in patients with glioblastoma. Anticancer Res. 2017;37:2159-71.

36. Munn DH, Zhou M, Attwood JT, et al. Prevention of allogeneic fetal rejection by tryptophan catabolism. Science. 1998;281: 1191-3.

37. Mellor AL, Baban B, Chandler P, et al. Cutting edge: Induced indoleamine 2,3 dioxygenase expression in dendritic cell subsets suppresses T cell clonal expansion. J Immunol. 2003;171:1652-5.

38. Szanto S, Koreny T, Mikecz K, Glant TT, Szekanecz Z, Varga J. Inhibition of indoleamine 2,3-dioxygenase-mediated tryptophan 
catabolism accelerates collagen-induced arthritis in mice. Arthritis Res Ther. 2007;9:R50.

39. Gurtner GJ, Newberry RD, Schloemann SR, McDonald KG, Stenson WF. Inhibition of indoleamine 2,3-dioxygenase augments trinitrobenzene sulfonic acid colitis in mice. Gastroenterology. 2003; 125:1762-73

40. Fallarino F, Volpi C, Zelante T, et al. IDO mediates TLR9-driven protection from experimental autoimmune diabetes. J Immunol. 2009;183:6303-12.

41. Yan Y, Zhang GX, Gran B, et al. IDO upregulates regulatory $\mathrm{T}$ cells via tryptophan catabolite and suppresses encephalitogenic $\mathrm{T}$ cell responses in experimental autoimmune encephalomyelitis. $\mathbf{J}$ Immunol. 2010;185:5953-61.

42. Heng B, Lim CK, Lovejoy DB, Bessede A, Gluch L, Guillemin GJ. Understanding the role of the kynurenine pathway in human breast cancer immunobiology. Oncotarget. 2016;7:6506-20.

43. Muller AJ, DuHadaway JB, Donover PS, Sutanto-Ward E, Prendergast GC. Inhibition of indoleamine 2,3-dioxygenase, an immunoregulatory target of the cancer suppression gene Bin1, potentiates cancer chemotherapy. Nat Med. 2005;11:312-9.

44. Ino K, Yamamoto E, Shibata K, et al. Inverse correlation between tumoral indoleamine 2,3-dioxygenase expression and tumorinfiltrating lymphocytes in endometrial cancer: Its association with disease progression and survival. Clin Cancer Res. 2008;14:2310-7.

45. Ino $\mathrm{K}$, Yoshida $\mathrm{N}$, Kajiyama $\mathrm{H}$, et al. Indoleamine 2,3-dioxygenase is a novel prognostic indicator for endometrial cancer. Br J Cancer. 2006;95:1555-61.

46. Liu J, Liu Y, Wang W, Wang C, Che Y. Expression of immune checkpoint molecules in endometrial carcinoma. Exp Ther Med. 2015;10:1947-52.

47. Strickland KC, Howitt BE, Shukla SA, et al. Association and prognostic significance of BRCA1/2-mutation status with neoantigen load, number of tumor-infiltrating lymphocytes and expression of PD-1/PD-L1 in high grade serous ovarian cancer. Oncotarget. 2016;7:13587-98.

48. Joneja U, Vranic S, Swensen J, et al. Comprehensive profiling of metaplastic breast carcinomas reveals frequent overexpression of programmed death-ligand 1. J Clin Pathol. 2016;70:255-9.

49. Thompson E, Taube JM, Elwood H, et al. The immune microenvironment of breast ductal carcinoma in situ. Mod Pathol. 2016;29:249-58.

50. Mansfield AS, Heikkila PS, Vaara AT, von Smitten KA, Vakkila JM, Leidenius MH. Simultaneous Foxp3 and IDO expression is associated with sentinel lymph node metastases in breast cancer. BMC Cancer. 2009;9:231.

51. Soliman HH, Minton SE, Han HS, et al. A phase I study of indoximod in patients with advanced malignancies. Oncotarget. 2016;7:22928-38.

52. Soliman HH, Jackson E, Neuger T, et al. A first in man phase I trial of the oral immunomodulator, indoximod, combined with docetaxel in patients with metastatic solid tumors. Oncotarget. 2014;5:8136-46.

53. Beatty GL, O’Dwyer PJ, Clark J, et al. First-in-human phase I study of the oral inhibitor of indoleamine 2,3-dioxygenase-1 epacadostat (INCB024360) in patients with advanced solid malignancies. Clin Cancer Res. 2017;23:3269-76.

54. Soliman H, Minton SE, Ismail-Khan R, et al. A phase 2 study of ad.p53 DC vaccine in combination with indoximod in metastatic solid tumors. J Clin Oncol. 2017;32(15_suppl).

55. Spira A, Hamid O, Bauer T, Borges VF, Wasser JS, Smith DC. Efficacy/safety of epacadostat plus pembrolizumab in triplenegative breast cancer and ovarian cancer: Phase I/II ECHO-202 study. J Clin Oncol. 2017;35(15_suppl):1103. 